\title{
Technologie, Organisation, Qualifikation
}

\author{
Ernst Hartmann und Steffen Wischmann
}

\subsection{Einleitung}

In diesem Band werden neun konkrete Beispiele von Arbeitssystemen beschrieben, die mit Techniken und Instrumenten der Industrie 4.0 gestaltet wurden (Teil 2 dieses Bandes). Weitere Beiträge thematisieren querschnittliche Aspekte wie:

- arbeitsplatznahes Beschreibungsmodell der „Arbeitswelt Industrie 4.0“ (Bauer et al. in diesem Band)

- Gerechtigkeit in flexiblen Arbeits- und Managementprozessen (Engels et al., in diesem Band)

- lernförderliche Arbeitsorganisation (Mühlbradt et. al., in diesem Band)

- den Einfluss des demographischen Wandels (Apt \& Bovenschulte in diesem Band)

- Datenverarbeitungsinfrastrukturen für zukünftige Entscheidungen (Meyer et al. in diesem Band)

- ein Leitbild für die Gestaltung soziotechnischer Systeme (Hirsch-Kreinsen et al. in diesem Band)

Für den Ausblick in diesem letzten Kapitel sollen die drei wesentlichen Aspekte der Arbeitswelt 4.0 - Technologie, Organisation und Qualifikation - im Zusammenhang und in einer zukunftsgerichteten Perspektive betrachtet werden.

E. Hartmann $(\bowtie)$

Institut für Innovation und Technik, Steinplatz 1, 10623 Berlin, Deutschland

e-mail: hartmann@iit-berlin.de

S. Wischmann

e-mail: wischmann@iit-berlin.de 
Dieser Dreiklang aus Technologie, Organisation und Qualifikation entspricht dem in der Arbeitsforschung seit langer Zeit etablierten Konzept des soziotechnischen Systems, das auch zur Beschreibung und Analyse von konkreten Arbeitssystemen verwendet wird (Trist und Bamforth 1951; Ulich 2005; Strohm und Ulich 2011; Hartmann 2005).

\title{
17.2 Was können wir über zukünftige Technologie, Organisation und Qualifikationen wissen?
}

\subsection{1 Überblick - ein methodischer Vorschlag}

Der konzeptionelle Fahrplan dieses Kapitels orientiert sich an einem methodischen Vorschlag zur Qualifikationsbedarfsprognose aufbauend auf Technologieroadmaps und Organisationsszenarien (Hartmann und Bovenschulte 2013).

In einem ersten Schritt werden Technologieroadmaps betrachtet, welche die zukünftige Verfügbarkeit von Technologien auf einem Zeitstrahl abbilden. Dabei können sowohl vorhandene Roadmaps interpretiert als euch neue Roadmaps erstellt werden. Solche Roadmaps werden in einzelnen Unternehmen erstellt. Diese Dokumente sind allerdings oft nicht frei verfügbar. Besser verfügbare Roadmaps - oder das zur Erstellung notwendige Wissen findet man auch bei Akteuren der Technologie- und Forschungsförderung, wie etwa Projektträgern und ähnlichen Agenturen oder auch bei den Europäischen Technologieplattformen, ${ }^{1}$ in denen Wirtschaft, Wissenschaft und intermediäre Organisationen zusammenarbeiten.

Solche Dokumente geben Aufschluss über entstehende technische Möglichkeiten. Zwingende Folgen für Organisation und Technik - im Sinne eines technologischen Determinismus - ergeben sich daraus allerdings nicht (Kärcher 2015). Deshalb werden in einem zweiten Schritt Organisationsszenarien beschrieben (Abb. 17.1). Sie stellen Möglichkeiten

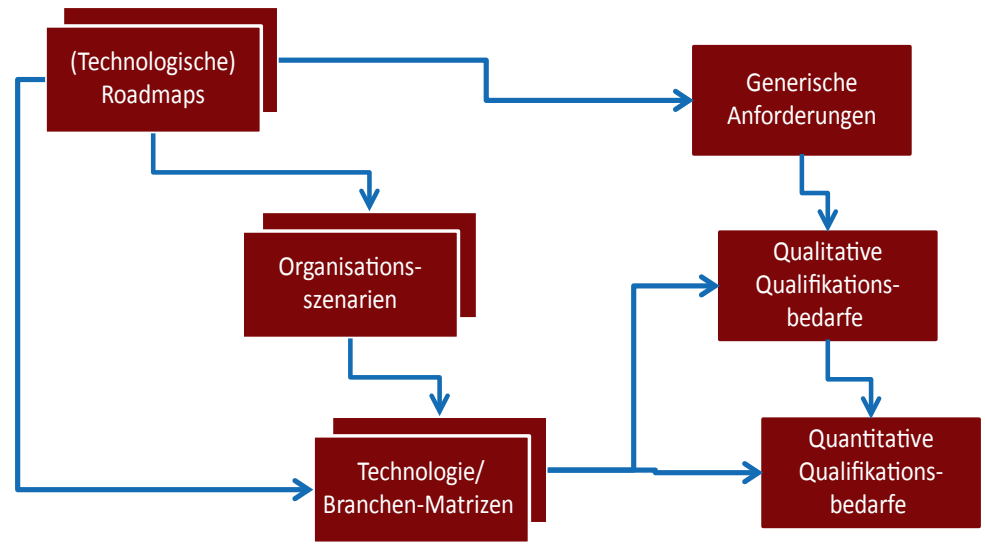

\begin{abstract}
Abb. 17.1 Vorschlag eines methodologischen Rahmens für die Qualifikationsbedarfsprognose (Hartmann und Bovenschulte 2013)
\end{abstract}

\footnotetext{
${ }^{1}$ http://ec.europa.eu/research/innovation-union/index_en.cfm?pg=etp
} 
zukünftiger Betriebs- und Arbeitsorganisation dar. Diese Organisationsparadigmen haben in der Regel den stärksten Einfluss auf die Arbeitswelt, einen stärkeren als die Technologie (Henning et al. 1994; Strohm und Ulich 2011; Brandt et al. 1999).

Auch die globale Kenntnis technologischer Entwicklungen und organisationaler Modelle reicht allein nicht aus. In unterschiedlichen Branchen werden sich Technologien unterschiedlich auswirken, auch die vorherrschenden Organisationsmodelle werden unterschiedlich sein. In Technologie/Branchen-Matrizen können diese Unterschiede abgebildet werden (Abb. 17.5). Auf dieser Spezifikationsebene - Technologien und Organisationsmodelle in Branchen können schließlich qualitativ und quantitativ Qualifikationsbedarfe bestimmt werden.

\subsubsection{Technologie-Roadmaps identifizieren und nutzen}

Es finden sich verschiedene Quellen für Technologie-Roadmaps - und ebenso vielfältige Methoden der Beschreibung und Strukturierung von Roadmaps. Im Folgenden werden einige dieser Quellen und Formate im Hinblick auf Industrie 4.0 vorgestellt.

Eine besondere Methode des Roadmapping ist das Visual Roadmapping (Kind et al. 2011). Mit der Methode kann das komplexe Wechselspiel des zu untersuchenden (technischen) Themas mit äußeren Einflussfaktoren im Hinblick auf zukünftige Entwicklung analysiert werden. Zu diesem Zweck werden in der Visual Roadmap vier Dimensionen abgebildet:

- sozio-ökonomische Einflussfaktoren (rechtliche, ökonomische, gesellschaftliche Rahmenbedingungen, Phänomene und Voraussetzungen)

- Enabling Technologies (wissenschaftlich-technische Voraussetzungen für das jeweilige Thema)

- Entwicklung des zu betrachtenden Themas (zentrale Aspekte und Meilensteine, die bei der Entwicklung des Themas von Bedeutung sind)

- Auswirkungen (ökonomische und gesellschaftliche Auswirkungen, aber auch neue Produkt- und Dienstleistungsangebote etc.)

Die Genese der Roadmap ist ein Experten basiertes Verfahren, das mit einzelnen Fachleuten oder in Gruppen von bis zu 10 Personen durchgeführt werden kann. Beginnend mit einer leeren Matrix (die vier Dimensionen versus die Zeitachse mit einem Horizont von etwa 10 bis 15 Jahren in die Zukunft), werden die Experten gebeten, das Thema zu erörtern und einzelne Aspekte/Kernfragen der zukünftigen Entwicklung in Bezug auf die vier Dimensionen zu identifizieren. Diese Aspekte und Fragen werden auf „Ereigniskarten“ notiert und in der Matrix (Dimension und Zeitpunkt) positioniert. Schritt für Schritt wird die Matrix mit weiteren Karten gefüllt, während bestehende Karten neu arrangiert oder weiter differenziert/aufgeschlüsselt werden können (z. B., wenn ein Aspekt in zwei Teilaspekte geteilt werden soll). Nach der abschließenden Anordnung der Karten werden prominente Verbindungen und Abhängigkeiten - vor allem dimensionsübergreifende - durch Linien und Pfeile hervorgehoben.

Mit dem beschriebenen Verfahren kann auch implizites Wissen von Experten verfügbar gemacht und anhand der vier Dimensionen klar strukturiert werden. 
Abb. 17.2 zeigt ein visuelles Roadmap zur Zukunft der digitalen Arbeit in der Produktion; sie wurde im Herbst 2015 im Rahmen eines Expertenworkshops für das Bundesministerium für Arbeit und Soziales erstellt. Auch wenn hier themenbedingt von der oben beschriebenen Struktur der vier Dimensionen etwas abgewichen wurde, wird doch die Einordnung der technologischen Entwicklung in einen systemischen Zusammenhang auch hier sehr deutlich.

Im Hinblick auf die Arbeitswelt der Zukunft waren den Experten folgende technologische Aspekte besonders wichtig:

- Mensch-Maschine-Teams, in denen Aufgaben kollaborativ und flexibel zwischen Menschen und Maschinen geteilt und organisiert werden

- Assistenzsysteme bis hin zu Tutorsystemen, die Menschen im Lernen unterstützen können

- generell Methoden und Gestaltungslösungen zur Minderung oder Vermeidung von Automationsdilemmata, die dadurch entstehen, dass der Mensch durch Automaten aus der aktiven Kontrolle des Systems verdrängt wird (Bainbridge 1983) ${ }^{2}$

Eine große Unsicherheit herrschte hinsichtlich zukünftiger industrieller Organisationssysteme. Die Ausweitung des bestehenden Konzepts „Ganzheitlicher Produktionssysteme“ $(\mathrm{GPS})^{3}$ auf die Bedingungen der Industrie 4.0 ist dabei nur eine Möglichkeit. Weiterhin erlaubt das Konzept der GPS auch erhebliche Variationen der tatsächlich wirksamen Arbeitsorganisation, die noch dazu oftmals auch innerhalb eines Unternehmens, selbst innerhalb eines einzelnen Betriebes variiert. Hier besteht noch erheblicher Forschungsund Datengewinnungsbedarf.

\subsubsection{Roadmaps Europäischer Technologieplattformen}

Europäische Technologieplattformen sind Bündnisse von Partnern aus Wirtschaft und Industrie, die sich in Europäischen Forschungsprogrammen engagieren. Im Hinblick auf Industrie 4.0 sind insbesondere folgende ETP relevant:

- European Technology Platform on Smart Systems Integration (EpoSS) ${ }^{4}$

- European Technology Platform Manufuture, ${ }^{5}$ sowie die von Manufuture mit initiierte PublicPrivate-Partnership "European Factories of the Future Research Association" (EFFRA) ${ }^{6}$

- euRobotics als Nachfolgeorganisation der European Robotics Technology Platform $(\text { EUROP })^{7}$ mit der PPP SPARC ${ }^{8}$

\footnotetext{
${ }^{2}$ Eine etwas ausführlichere Darstellung der Automationsdilemmata findet sich im Beitrag von Ernst Hartmann in diesem Band zu den gestaltbaren Dimensionen der Arbeitssysteme im Abschnitt „Kontrolle“.

${ }^{3}$ vgl. VDI-Richtlinie: VDI 2870 Blatt 1 Ganzheitliche Produktionssysteme - Grundlagen, Einführung und Bewertung

${ }^{4}$ http://www.smart-systems-integration.org/public

${ }^{5}$ http://www.manufuture.org/manufacturing/

${ }^{6} \mathrm{http}: / / \mathrm{www} . e f f r a . e u$

${ }^{7}$ https://eu-robotics.net/

${ }^{8} \mathrm{http} / / /$ sparc-robotics.eu/about/
} 


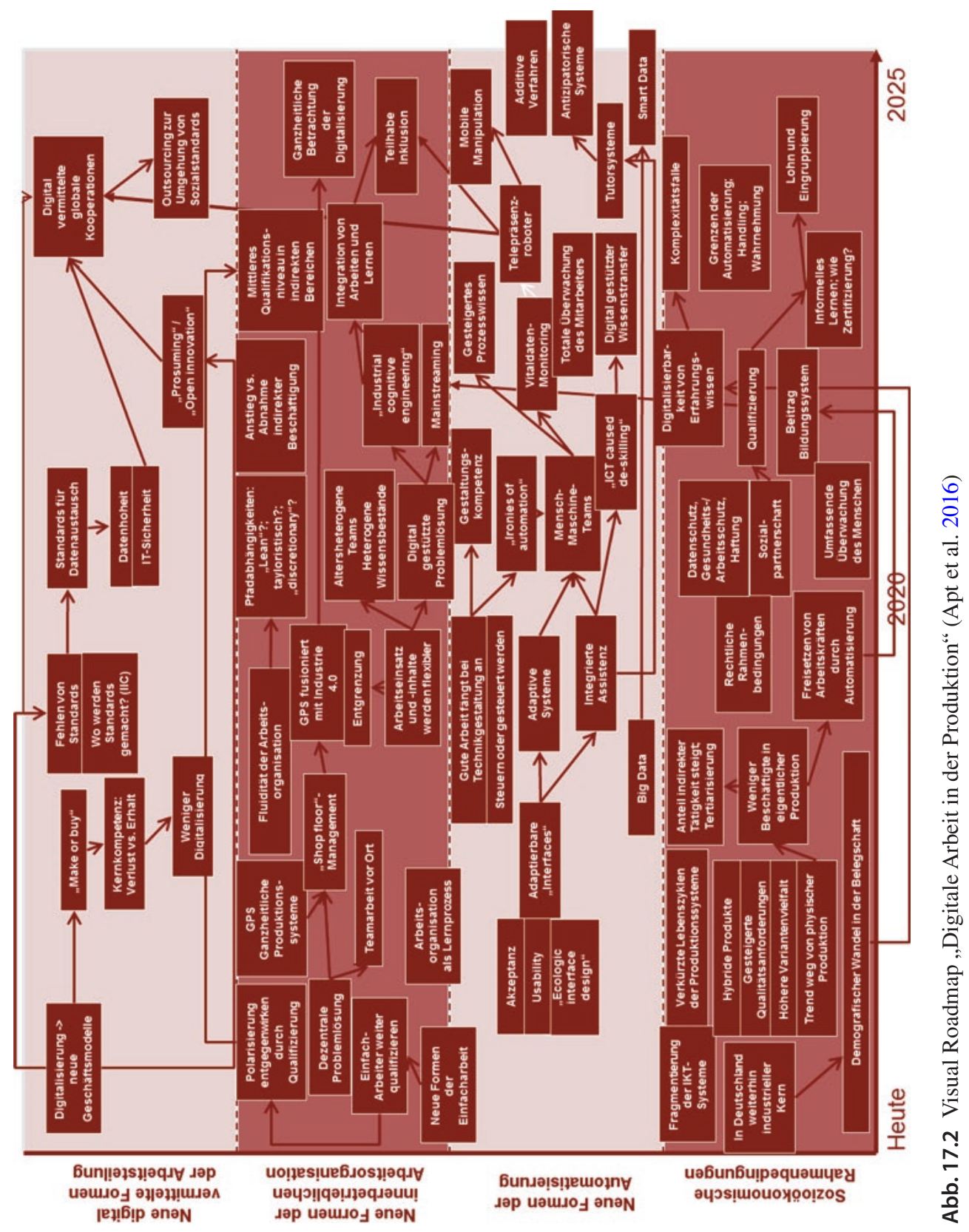


Die jüngste Überarbeitung der EPoSS-Roadmap wurde auch abgeglichen mit den Manufuture/EFFRA Roadmaps (Abb. 17.3).

Beispielhaft können folgende technologischen Aspekte hervorgehoben werden:

- selbstkonfigurierende und -organisierende Produktionssysteme

- ,selbstheilende“ Produktionssysteme

- Integration biotechnologischer Komponenten

- maschinelle Kognition und prädiktive Steuerung

Etliche Aspekte überlappen auch mit der oben (Abb. 17.2) dargestellten Roadmap aus der Foresight-Studie für das BMAS.

Eine weitere interessante und relevante Roadmap ist die Robotics 2020 Multi-Annual Roadmap von euRobotics/SPARC aus dem Jahr 2016. Die zentrale Vision zukünftiger Roboter in der industriellen Produktion wird dort wie folgt beschrieben:

„The key product vision in manufacturing is of a robot able to safely operate in an semistructured environment in physical collaboration with human operators. To be configured using intuitive interfaces by operators rather than by specialised programmers. These new systems need to have flexibility not only with respect to the user interface but also with respect to the task. Generic grippers, gripping strategies and planning and control systems able to adapt to different optimisation parameters, and to dynamic environments without compromising safety.

This vision involves the integration of a much broader range of sensing and interpretation technologies with advanced systems development and human robot interaction technologies.“ (Robotics 2020 Multi-Annual Roadmap, S. 279)

Kernaspekte sind die sichere Kooperation mit Menschen, die intuitive Kooperation/Programmierung/Konfiguration durch den unmittelbaren Operateur (nicht durch Spezialisten) sowie neue und flexible Greifer und Greifstrategien für neue Objektklassen (z. B. biegeschlaffe Objekte).

Dieser sehr knappe Überblick über Technologie-Roadmaps mag einen ersten Eindruck der unterschiedlichen Quellen und Formate geben. Im Folgenden soll dargestellt werden, wie diese Informationen als Grundlage einer Qualifikationsvorausschau verwendet werden können.

\subsubsection{Generische Anforderungen an Qualifikationen}

Eine integrative Betrachtung der verschiedenen Technologie-Roadmaps führt zu einigen generischen Anforderungen an Qualifikationen. Eine dieser Anforderungen betrifft die fortschreitende Konvergenz mechanischer, elektronischer und softwarebasierter Systeme, die sich über unterschiedliche Skalenniveaus (makro/meso/mikro) erstreckt.

\footnotetext{
${ }^{9}$ Robotics 2020 Multi-Annual Roadmap-For Robotics in Europe. Release B 03/12/2015. http://sparcrobotics.eu/wp-content/uploads/2014/05/H2020-Robotics-Multi-Annual-Roadmap-ICT-2016.pdf
} 


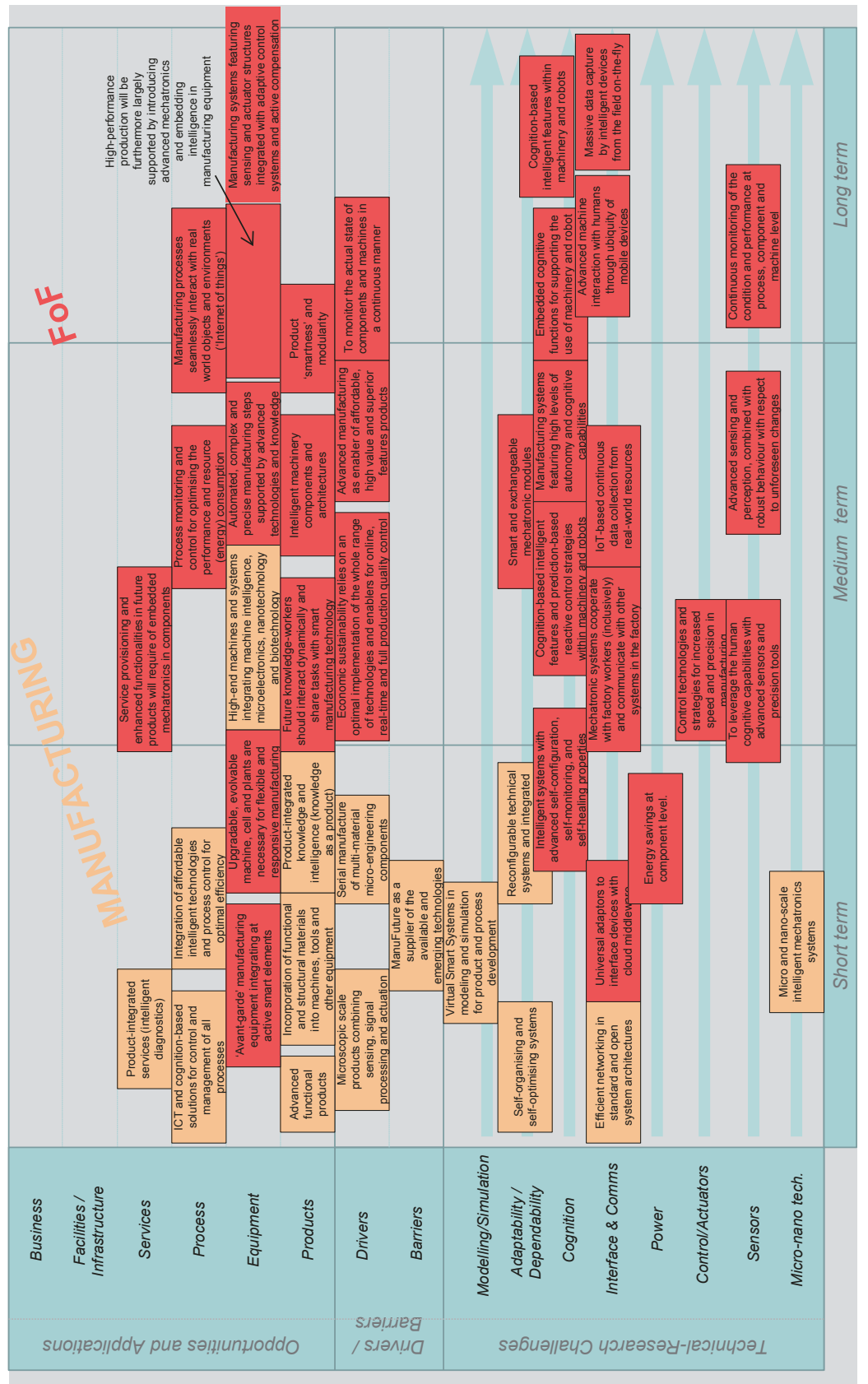

๓্口

है

त त

is

हี

ठ

.

bू 5

光

है ते

के छิ

클 छ

की के

ฮั

壱宁

巳

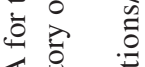

『苞

递

预 党

这害

일 है ठ

窇 苛

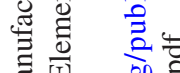

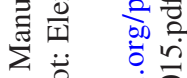

$\Xi$ 잉유

है ڤี

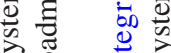

क $\stackrel{0}{\Xi}$ ते

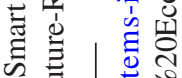

थ

记导 余号

클 究

苛㐫

\&

m.

들 苞

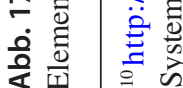


Ein besonders relevantes und dynamisches Technologiefeld scheint die Robotik zu sein. Wichtige Aspekte sind hier kooperierende Roboter und Fragen der ,weichen Automatisierung“ (z. B. inhärente Sicherheit durch weiche und flexible Aktuatoren - etwa „Elefantenrüssel“" robotischer Systeme). Weiterhin könnte die Bionik in Zukunft eine wichtigere Rolle spielen für die Entwicklung von robotischen Systemen mit „nahezu menschlichen“ Fähigkeiten der Wahrnehmung, Kognition und Motorik.

Die flexible Arbeitsteilung zwischen Mensch und - kooperierendem - Roboter wirft neue Sicherheitsfragen auf. Wenn es keine festgelegten Arbeitsabläufe gibt, muss jede Situation im Arbeitsprozess selbst neu unter Sicherheitsaspekten beurteilt werden.

\subsubsection{Qualitative Qualifikationsbedarfe: Erste Hypothesen}

Im Hinblick auf die oben beschriebenen generischen Anforderungen können einige erste Hypothesen hinsichtlich des qualitativen Qualifikationsbedarfs formuliert werden. Generell zeichnen sich die Technologien der Industrie 4.0 durch eine neue Stufe der Integration von mechanischen, elektronischen und informatischen Komponenten aus, letztere einschließlich Elementen der Künstlichen Intelligenz (KI). Für den Bereich der beruflichen Bildung muss dies nicht unbedingt zu neuen Berufsbildern führen. Es ist - zumindest als mittelfristige Vision - auch eine stärkere Berücksichtigung von Inhalten der industriellen Informatik in der Ausbildung etwa von Mechatronikern oder Produktionstechnologen denkbar. Dies könnte zu standardisierten Zusatzqualifikationen führen, die auf eine spätere Weiterbildung angerechnet werden könnten. Speziell im Bereich der Weiterbildung, aufbauend auf bewährten Berufsprofilen, sind viele Industrie-4.0-affine Elemente denkbar, nicht nur im Feld der industriellen Informatik, sondern auch der Sensorik oder der Robotik.

Als zukünftiges hochschulisches Bildungsangebot ist „Industrielle Kognitionswissenschaft" denkbar, etwa als weiterbildendes Zertifikats- oder Masterprogramm für Maschinenbau- und Elektroingenieure. Zentrale Inhalte könnten hier verteilte Sensor-/Aktornetze, Robotik, Wahrnehmung (z. B. 3D-Sehen) und Kognition (z. B. Handlungsplanung, Kooperation, Schwarmintelligenz) sein.

In ähnlicher Weise ist eine Spezialisierung in „Automationsbionik“ denkbar, die sich ebenfalls auf Robotik bezieht mit Akzenten in der Aktorik (z. B. künstliche Muskeln, Gliedmaßen und Organe) und ebenfalls Aspekten der Wahrnehmung und Kognition aus einer eher biologischen Perspektive.

\subsubsection{Organisationsszenarien}

Modelle der Betriebs- und Arbeitsorganisation haben generell den größten Einfluss auf die Arbeitswelt der Zukunft: Die Arbeitsorganisation bestimmt, wer welche Aufgaben zugewiesen bekommt und wie groß die Handlungsspielräume bei der Bearbeitung dieser Aufgaben sind (Hacker 2005). 
Werkzeugszenario

Automatisierungsszenario

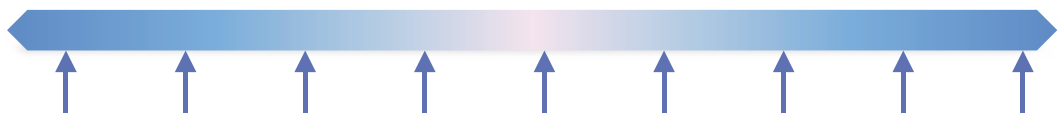

Aufwertung von Qualifikationen

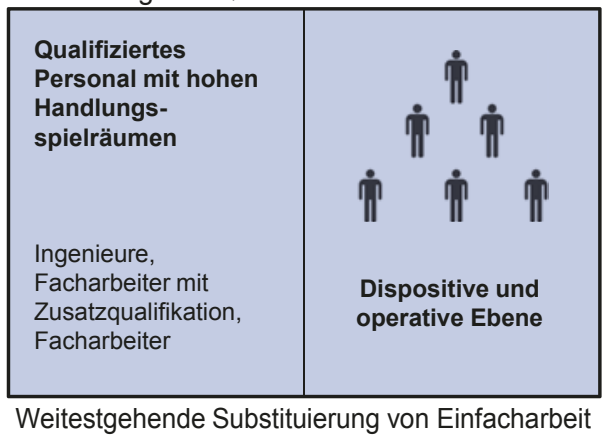

Polarisierung von Qualifikationen

Abb. 17.4 Organisationsszenarien und ihre Auswirkungen auf Qualifikationsstrukturen (eigene Darstellung des iit nach (Windelband und Dworschak 2015; Hirsch-Kreinsen 2015))

Unterschiedliche Organisationszenarien werden oft als zwei diametral gegenüberliegende Pole beschrieben (Abb. 17.4), von denen einer, in grober Näherung, eher „Menschorientiert“" und einer eher „Technik-orientiert“ ist. Aktuell diskutierte Begriffe beziehen sich etwa auf eine Werkzeugszenario, das den Menschen in den Mittelpunkt stellt und ihm die Technik als Werkzeug an die Hand gibt, gegenüber einem Automatisierungsszenario (Windelband und Dworschak 2015).

Oder es wird eine integrative, auf eher homogene und insgesamt stark ausgeprägte Qualifikationen setzende „Schwarm-Organisation“ einer polarisierten Organisation gegenübergestellt, in der sich nur noch Niedrig- und Hochqualifizierte finden, ohne mittlere Qualifikationen (insbes. Facharbeiter) (Hirsch-Kreinsen 2015). Aus innovationsökonomischer Perspektive wird auch zwischen vier Organisationstypen (discretionary learning (DL), lean, taylorist und traditional) unterschieden, die u. a. verschiedene Grade der Lernintensität aufweisen (Lorenz und Valeyre 2005; Lorenz 2015).

Die durch die Organisationsmodelle induzierten unterschiedlichen Qualifikationsbedarfe sollen im Folgenden noch anhand zweier Textfragmenten aus zwei Szenarien illustriert werden. Die Beispiele stammen aus einer Studie des iit im Kontext des DGB-Technologiedialogs (Hartmann 2009). Neben faktenbasiertem, analytischem Material enthält diese Studie auch anschauliche Szenarien; daraus stammt der folgende fiktive Dialog zweier Betriebsräte um das Jahr 2020. Der erste Betriebsrat schildert diese Situation:

Ich erklär' das mal am Beispiel der Einrichter. Die haben jetzt alle Daten-Brillen auf. Wenn irgendwo was gemacht werden muss, kriegen sie das in die Brille eingeblendet, also zum 
Beispiel ,ab nach Halle 13, zur Birkenbach-Maschine'. Da stehen dann schon alle Werkzeuge und Vorrichtungen bereit, die sie brauchen, auf automatischen Wagen. Die müssen dann auch gar nicht mehr groß nachdenken, was sie zuerst machen sollen, denn die einzelnen Werkzeuge leuchten farbig auf - in der Brille natürlich, nicht in echt - wenn sie dran sind. Wenn dazu noch Infos nötig sind, leuchten die auch in der Brille auf.

Eine Kollegin aus einem anderen Betrieb stellt dem dies gegenüber:

Spezielle Einrichter haben wir ja gar nicht, alle im Produktionsteam können die Maschinen auch einrichten, auch zu einem guten Teil warten. Weil wir diese komplette Vernetzung haben, können wir an unseren Info-Terminals ${ }^{11}$ immer genau sehen, wie der Zustand in der Fertigung ist. Und noch besser: Auch, wie er demnächst sein wird, weil wir zum Beispiel immer sehen können, ob unsere Nachschubteile gerade auf der Autobahn stehen oder schon auf den Hof rollen. So können wir unsere Arbeit prima organisieren und abstimmen.

Aus diesen Beispielen sollte ersichtlich sein, dass ähnliche oder sogar identische Technologien in Abhängigkeit von ihrer Einbettung in bestimmte Organisationsformen sehr unterschiedliche Qualifikationsbedarfe begründen können.

\subsubsection{Technologie/Branchen-Matrizen}

Schließlich müssen bei der Qualifikationsbedarfsanalyse die Effekte in unterschiedlichen Branchen differenziert betrachtet werden (Abb. 17.5). Dies mag sich zunächst auf unterschiedliche Organisationsparadigmen beziehen, die in unterschiedlichen Branchen

Abb. 17.5 Technologie/Branchen-Matrix in Abhängigkeit von Beschäftigungssegmenten (Quelle: modifiziert aus (Hartmann und Bovenschulte 2013))

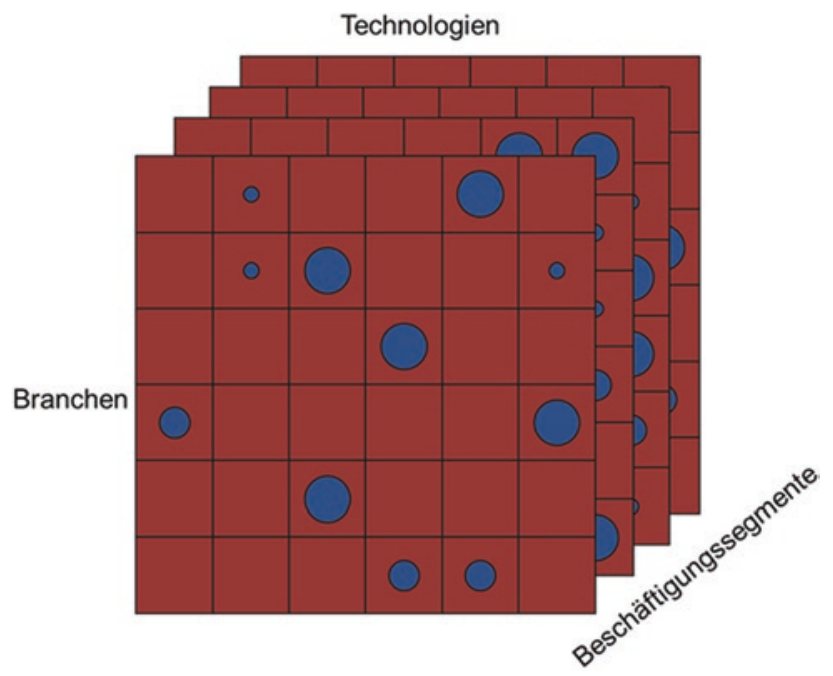

${ }^{11}$ Hier merkt man, wie alt ein Text aus dem Jahre 2009 wirken kann. Heute würde man eher an mobile Endgeräte denken. 
unterschiedlich verbreitet sind. Weiterhin werden in - bezogen auf die jeweilige Technologie - herstellenden und anwendenden Branchen verschiedene Bedarfe zu erwarten sein.

Innerhalb der Branchen werden die Effekte wiederum nach Arbeitskräftesegmenten variieren (z. B. Konstruktion gegenüber Produktion, beruflich gegenüber hochschulisch gebildetem Personal).

Abb. 17.6 zeigt beispielhaft eine Technologie/Branchen-Matrix für einige Technologien der Industrie 4.0. Hier sind die Effekte noch nicht auf einzelne Beschäftigtengruppen heruntergebrochen worden, wie es für eine Qualifikationsbedarfsanalyse notwendig wäre.

\subsection{Fazit}

Technologie, Organisation und Qualifikation sind die zentralen Parameter der Arbeitswelt. Anhand technologischer Roadmaps können zukünftige technische Entwicklungen hinsichtlich ihrer Qualität und ihrer zeitlichen Dynamik (erste Verfügbarkeit, weitere Entwicklung) abgeschätzt werden. Daraus ergeben sich auch erste, grobe Hinweise auf generische Qualifikationsbedarfe. Hinsichtlich Industrie 4.0 sind diese etwa in den Bereichen industrielle Informatik, Künstliche Intelligenz, Robotik und Bionik zu vermuten.

Konkrete, zuverlässige Prognosen zur Ausformungen zukünftiger Produktionsarbeit lassen sich aktuell nicht finden. Dies betrifft insbesondere die Facharbeit, die an sich über gute Potenziale für eine Qualifikationsaufwertung verfügt. Einstimmigkeit herrscht lediglich hinsichtlich zweier zu erwartender Trends. Der industriellen Einfacharbeit (bspw. durch Angelernte) werden schlechte Entwicklungschancen zugerechnet. Ihre Rolle wird unter Umständen hauptsächlich in der Besetzung von Rationalisierungsnischen bestehen. Für Hochqualifizierte mit akademischen Abschlüssen bieten sich unabhängig von den sich zukünftig entfalteten Organisationsszenarien durchweg sehr gute Entwicklungsperspektiven. Gefordert sind dabei allerdings erhebliche Anstrengungen, um den neuen Qualifikationsanforderungen und gesteigerten Flexibilitätsansprüchen gerecht zu werden.

Angesichts der hier angerissenen Diskussion lässt sich die These ableiten, dass die technologischen Einsatzszenarien durchaus mit den Qualifikationsszenarien gekoppelt sind (Abb. 17.4). Es ist davon auszugehen, dass das eine Ende des Spektrums, der Einsatz von neuen Technologien als Werkzeuge, eine Aufwertung von Qualifikationen fördert, während das andere Ende, die Erreichung eines möglichst hohen Automatisierungsgrades, eine Polarisierung von Qualifikationen verstärkt.

Die skizzierten Zusammenhänge erlauben auf der aktuellen Wissensbasis keine eindeutige Abschätzung über die Folgen der Digitalisierung für die Arbeit. Welche Arbeitsorganisation und -formen sich durchsetzen werden, wird sehr stark von unternehmerischen Entscheidungsprozessen abhängen (Kärcher 2015). Diese Wahlfreiheit ermöglicht hohe Gestaltungsmöglichkeiten. Es sollte jedoch bedacht werden, dass der Einsatz digitaler Technologien entweder als unterstützendes Werkzeug oder als reine Automatisierungsmöglichkeit, diese Wahlfreiheit unter Umständen von Beginn an einschränken kann. D. h. für die einzelnen Unternehmen kann es durchaus sinnvoll sein, sich zuerst auf die gewünschten arbeitsorganisatorischen Gestaltungsziele und Qualifikationsentwicklungen 


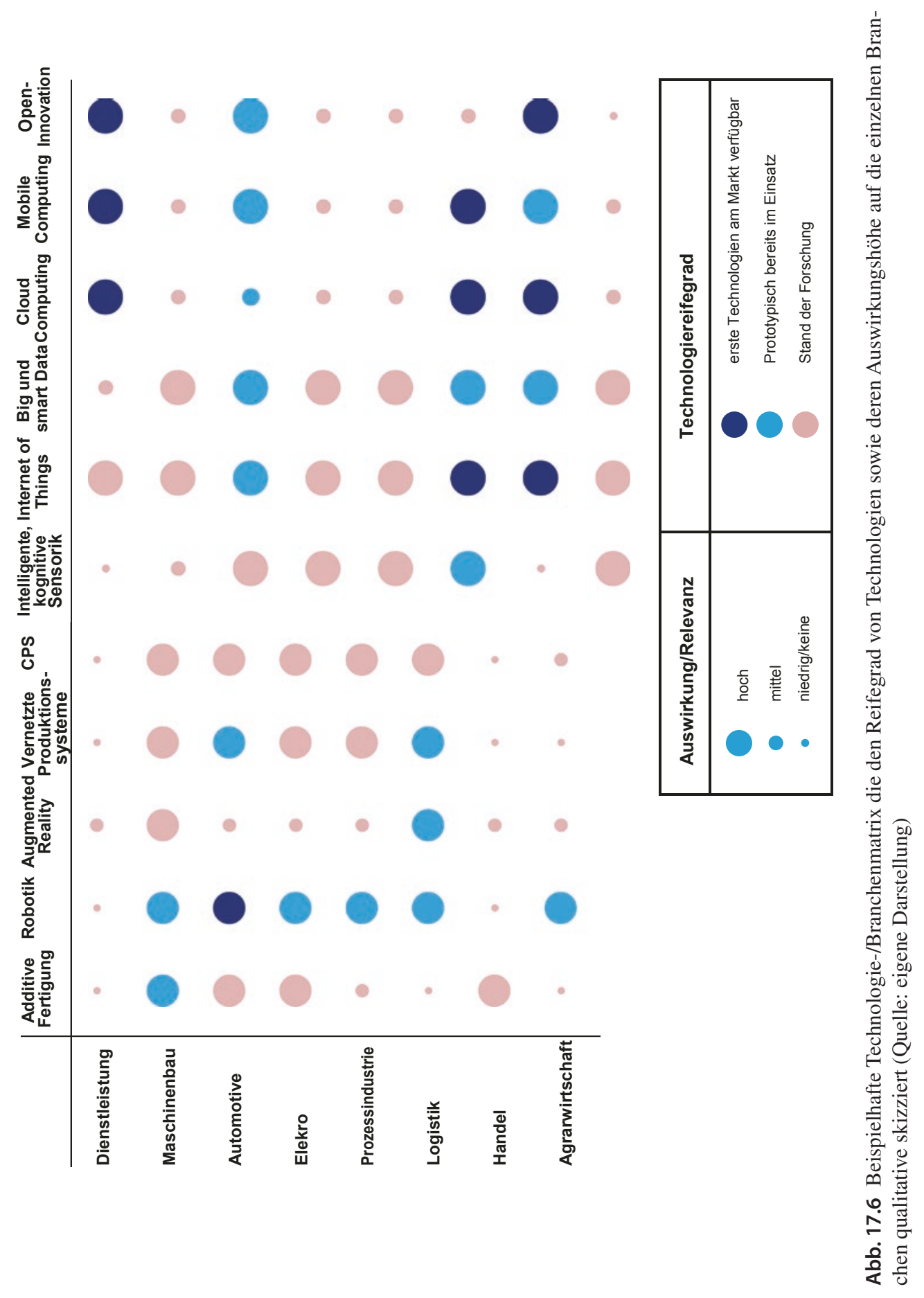


zu konzentrieren und dann die entsprechenden Technologien auszuwählen und zum Einsatz zu bringen. Hierbei ist anzumerken, dass es keinen allgemeinen Technikdeterminismus gibt. Dieselbe Technologie kann sich an unterschiedlichen Orten des skizzierten Spektrums manifestieren.

\section{Literatur}

Apt, W., Bovenschulte, M., Hartmann, E. A., \& Wischmann, S. (2016). Foresightstudie 'Digitale Arbeit. Bundesministerium für Arbeit und Soziales. Berlin (Forschungsbericht, 463). Online verfügbar unter http://www.bmas.de/SharedDocs/Downloads/DE/PDF-Publikationen/Forschungsberichte/f463-digitale-arbeitswelt.pdf?_blob=publicationFile\&v=2.

Bainbridge, L. (1983). Ironies of automation. Automatica, 19(6), 775-779. https://doi. org/10.1016/0005-1098(83)90046-8.

Brandt, D., Hartmann, E., Sander, C., \& Strina, G. (1999). Designing and simulating sociotechnical systems: concepts and strategies. Human Factors and Ergonomics in Manufacturing \& Service Industries, 9(3), 245-252. Zugegriffen:15 Juli 2013.

Hacker, W. (2005). Allgemeine Arbeitspsychologie. Psychische Regulation von Wissens-, Denkund körperlicher Arbeit. 2., vollst. überarb. und erg. Aufl. Bern: Huber (Schriften zur Arbeitspsychologie, 58).

Hartmann, E. A. (2005). Arbeitssysteme und Arbeitsprozesse. Zürich: vdf, Hochschulverlag.

Hartmann, E. A. (2009). Internet der Dinge-Technologien im Anwendungsfeld ,Produktion - Fertigungsplanung ‘. In A. Botthof \& M. Bovenschulte (Hrsg.), Das, Internet der Dinge'. Die Informatisierung des Alltags und der Lebenswelt. Düsseldorf/Germany: Hans-Böckler-Stiftung (AP 176.

Hartmann, E. A., \& Bovenschulte, M. (2013): Skills needs analysis for "Industry 4.0" based on roadmaps for smart systems. In Using technology foresights for identifying future skills needs. Global Workshop Proceedings. Moskow: Skolkovo, International Labour Organization.

Henning, K., Volkholz, V., Risch, W., \& Hacker, W. (Hrsg.) (1994). Moderne LernZeiten. Berlin/ Heidelberg/New York: Springer.

Hirsch-Kreinsen, H. (2015). Entwicklungsperspektiven von Produktionsarbeit. In A. Botthof \& E. A. Hartmann (Hrsg.), Zukunft der Arbeit in Industrie 4.0. Berlin, Heidelberg: Springer.

Kärcher, B. (2015). Alternative Wege in die Industrie 4.0 - Möglichkeiten und Grenzen. In A. Botthof \& E. A. Hartmann (Hrsg.), Zukunft der Arbeit in Industrie 4.0. Berlin, Heidelberg: Springer.

Kind, S., Hartmann, E. A., \& Bovenschulte, M. (2011). Die Visual-Roadmapping-Methode für die Trendanalyse, das Roadmapping und die Visualisierung von Expertenwissen. Institut für Innovation und Technik (iit) (iit perspektive, 4). Online verfügbar unter http://www.iit-berlin.de/de/ publikationen/iit-perspektive-4.

Lorenz, E. (2015). Work organisation, forms of employee learning and labour market structure. Accounting for international differences in workplace innovation. Journal of the Knowledge Economy, 6(2), 437-466. https://doi.org/10.1007/s13132-014-0233-4..

Lorenz, E., \& Valeyre, A. (2005). Organisational innovation, human resource management and labour market structure. A comparison of the EU-15. Journal of Industrial Relations, 47(4), 424-442. https://doi.org/10.1111/j.1472-9296.2005.00183.x.

Strohm, O., \& Ulich, E. (2011). Unternehmen umfassend bewerten. In C. Meyn, G. Peter, U. Dechmann, A. Georg, \& O. Katenkamp (Hrsg.), Arbeitssituationsanalyse (S. 322-338). Wiesbaden: VS Verlag für Sozialwissenschaften. 
Trist, E., \& Bamforth, K. (1951). Some social and psychological consequences of the long wall method of coal getting. Human Relations, 4, 3-38.

Ulich, E. (2005). Arbeitspsychologie. 6., überarb. und erw. Aufl. Zürich: vdf Hochschulverl. an der ETH. Online verfügbar unter http://deposit.ddb.de/cgi-bin/dokserv?id=2646503\& prov=M\&dok_var=1\&dok_ext=htm.

Windelband, L., \& Dworschak, B. (2015). Arbeit und Kompetenzen in der Industrie 4.0. Anwendungsszenarien Instandhaltung und Leichtbaurobotik. In H. Hirsch-Kreinsen (Hrsg.), Digitalisierung industrieller Arbeit. Die Vision Industrie 4.0 und ihre sozialen Herausforderungen. [Elektronische Ressource]. Berlin: Nomos.

Open Access Dieses Kapitel wird unter der Creative Commons Namensnennung 4.0 International Lizenz (http://creativecommons.org/licenses/by/4.0/deed.de) veröffentlicht, welche die Nutzung, Vervielfältigung, Bearbeitung, Verbreitung und Wiedergabe in jeglichem Medium und Format erlaubt, sofern Sie den/die ursprünglichen Autor(en) und die Quelle ordnungsge-mäß nennen, einen Link zur Creative Commons Lizenz beifügen und angeben, ob Änderungen vorgenommen wurden.

Die in diesem Kapitel enthaltenen Bilder und sonstiges Drittmaterial unterliegen ebenfalls der genannten Creative Commons Lizenz, sofern sich aus der Abbildungslegende nichts anderes ergibt. Sofern das betreffende Material nicht unter der genannten Creative Commons Lizenz steht und die betreffende Handlung nicht nach gesetzlichen Vorschriften erlaubt ist, ist für die oben aufgeführten Weiterverwendungen des Materials die Einwilligung des jeweiligen Recht-einhabers einzuholen.

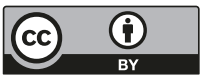

